\title{
THE SUPER-CONNECTIVITY OF KNESER GRAPHS
}

\author{
Gülnaz Boruzanli Ekinci \\ Department of Mathematics, Faculty of Science \\ Ege University, Bornova 35100, İzmir, Turkey \\ e-mail: gulnaz.boruzanli@ege.edu.tr \\ AND \\ John Baptist GaUCi \\ Department of Mathematics, Faculty of Science \\ University of Malta, Msida MSD2080, Malta \\ e-mail: john-baptist.gauci@um.edu.mt
}

\begin{abstract}
A vertex cut of a connected graph $G$ is a set of vertices whose deletion disconnects $G$. A connected graph $G$ is super-connected if the deletion of every minimum vertex cut of $G$ isolates a vertex. The super-connectivity is the size of the smallest vertex cut of $G$ such that each resultant component does not have an isolated vertex. The Kneser graph $K G(n, k)$ is the graph whose vertices are the $k$-subsets of $\{1,2, \ldots, n\}$ and two vertices are adjacent if the $k$-subsets are disjoint. We use Baranyai's Theorem on the decompositions of complete hypergraphs to show that the Kneser graph $K G(n, 2)$ are super-connected when $n \geq 5$ and that their super-connectivity is $\left(\begin{array}{l}n \\ 2\end{array}\right)-6$ when $n \geq 6$.
\end{abstract}

Keywords: connectivity, super-connectivity, Kneser graphs.

2010 Mathematics Subject Classification: 05C40, 94C15.

\section{REFERENCES}

[1] C. Balbuena, X. Marcote and P. García-Vázquez, On restricted connectivities of permutation graphs, Networks 45 (2005) 113-118. doi:10.1002/net.20056

[2] Zs. Baranyai, On the factorization of the complete uniform hypergraph, Colloq. Math. Soc. János Bolya 10 (1975) 91-108. 
[3] F.T. Boesch, Synthesis of reliable networks - a survey, IEEE Trans. Reliability 35 (1986) 240-246. doi:10.1109/TR.1986.4335424

[4] F.T. Boesch and R. Tindell, Circulants and their connectivities, J. Graph Theory 8 (1984) 487-499. doi:10.1002/jgt.3190080406

[5] G. Boruzanlı Ekinci and J.B. Gauci, On the reliability of generalized Petersen graphs, Discrete Appl. Math., in press. doi:10.1016/j.dam.2017.02.002

[6] G. Boruzanl Ekinci and A. Kırlangiç, Super connectivity of Kronecker product of complete bipartite graphs and complete graphs, Discrete Math. 339 (2016) 19501953. doi:10.1016/j.disc.2015.10.036

[7] B.-L. Chen and K.-W. Lih, Hamiltonian uniform subset graphs, J. Combin. Theory, Ser. B 42 (1987) 257-263. doi:10.1016/0095-8956(87)90044-X

[8] F. Harary, Conditional connectivity, Networks 13 (1983) 347-357. doi:10.1002/net.3230130303

[9] M.-C. Heydemann, Cayley graphs and interconnection networks, in: Graph Symmetry: Algebraic Methods and Applications, G. Hahn and G. Sabidussi (Ed(s)), (Springer, Dordrecht, 1997) 167-224. doi:10.1007/978-94-015-8937-6_5

[10] L.-H. Hsu and C.-K. Lin, Graph Theory and Interconnection Networks (CRC Press, 2008).

[11] M. Kneser, Aufgabe 360, Jahresber. Dtsch. Math.-Ver. 58 (1955) 27.

[12] M. Lü, C. Wu, G.-L. Chen and C. Lv, On super connectivity of Cartesian product graphs, Networks 52 (2008) 78-87. doi:10.1002/net.20224

[13] J.H. van Lint and R.M. Wilson, A Course in Combinatorics, 2nd edition (Cambridge University Press, Cambridge, 2001). doi:10.1017/CBO9780511987045

[14] M.E. Watkins, Connectivity of transitive graphs, J. Combin. Theory 8 (1970) 23-29. doi:10.1016/S0021-9800(70)80005-9

[15] W. Yang and J. Meng, Extraconnectivity of hypercubes, Appl. Math. Lett. 22 (2009) 887-891. doi:10.1016/j.aml.2008.07.016

[16] W. Yang and J. Meng, Extraconnectivity of hypercubes (II), Australas. J. Combin. 47 (2010) 189-195.

Received 10 October 2016 Revised 18 March 2017 Accepted 18 March 2017 